$$
\begin{array}{llll}
\lrcorner & \lrcorner & \lrcorner & \lrcorner
\end{array}
$$$$
\text { K3 surfaces with Picard rank } 20
$$$$
\text { Matthias Schütt }
$$$$
\lrcorner
$$$$
\rfloor
$$ 


\title{
K3 surfaces with Picard rank 20
}

\author{
Matthias Schütt
}

\begin{abstract}
We determine all complex K3 surfaces with Picard rank 20 over $\mathbb{Q}$. Here the Néron-Severi group has rank 20 and is generated by divisors which are defined over $\mathbb{Q}$. Our proof uses modularity, the Artin-Tate conjecture and class group theory. With different techniques, the result has been established by Elkies to show that Mordell-Weil rank 18 over $\mathbb{Q}$ is impossible for an elliptic K3 surface. We apply our methods to general singular K3 surfaces, that is, those with NéronSeveri group of rank 20 , but not necessarily generated by divisors over $\mathbb{Q}$.
\end{abstract}

\section{Introduction}

Complex K3 surfaces of geometric Picard number 20 are called singular since they involve no moduli. They share many properties with elliptic curves with complex multiplication (CM). For instance, they can always be defined over some number field. Moreover, over some finite extension of the number field, the $L$-series is given in terms of Hecke characters (see Theorem 29).

For singular K3 surfaces over $\mathbb{Q}$, Livné [1995] proved motivic modularity. However, this definition does not require that the Néron-Severi group be generated by divisors which are defined over $\mathbb{Q}$. We refer to this particular property as "Picard rank 20 over $\mathbb{Q} "$.

The motivation to study such K3 surfaces was the following: Shioda [1994] raised the question whether it was possible for an elliptic K3 surface to have Mordell-Weil rank 18 over $\mathbb{Q}$. One way to disprove this would have been to show that in general, K3 surfaces with Picard rank 20 over $\mathbb{Q}$ do not exist.

However, it turned out that there are such examples (see Examples 8, 9). Recently Elkies determined all these surfaces in terms of their transcendental lattices:

Theorem 1 [Elkies 2007]. Let $X$ be a K3 surface with Picard rank 20 over $\mathbb{Q}$. Then the transcendental lattice $T(X)$ is primitive of class number one.

MSC2000: primary 14J28; secondary 11F11, 11G15, 11G25, 11R29.

Keywords: singular K3 surface, Artin-Tate conjecture, complex multiplication, modular form, class group. 
Using sphere packings and gluing up to a Niemeier lattice, Elkies concluded that Mordell-Weil rank 18 over $\mathbb{Q}$ is impossible for an elliptic K3 surface.

Conversely, let $T(X)$ be primitive of class number one. Then the singular K3 surface $X$ with transcendental lattice $T(X)$ has a model with Picard rank 20 over $\mathbb{Q}$ (see Section 10).

In this paper, we present an alternative proof of Theorem 1 that we hope will be of independent interest. Our proof uses the following ingredients: modularity plus the classification of CM-forms in [Schütt 2009]; reduction and the Artin-Tate conjecture at split primes; and class group theory.

We then generalise our techniques to all singular K3 surfaces. We deduce the following obstruction to the field of definition:

Theorem 2. Let $L$ be a number field and $X$ a K3 surface of Picard rank 20 over $L$. Denote the discriminant of $X$ by $d<0$. Then $L(\sqrt{d})$ contains the ring class field $H(d)$.

This result enables us to give a direct proof of Shafarevich's finiteness theorem for singular K3 surfaces (Theorem 35). It is the only known obstruction for the field of definition of a singular K3 surface other than the result on the genus of $T(X)$ in [Schütt 2007b] (see (1) on next page and Lemma 34). In private correspondence, Elkies has informed me that his proof for Theorem 1 also generalises to Theorem 2 .

The paper is organised as follows: The next two sections recall the relevant facts about singular K3 surfaces and modularity. In Section 4 we give two explicit examples of K3 surfaces of Picard rank 20 over $\mathbb{Q}$. Section 5 introduces the main techniques to be used, particularly the Artin-Tate conjecture. The proof of Theorem 1 is presented in Sections 6 through 9. The converse statement of Theorem 1 is covered in Section 10. We continue with the classification of K3 surfaces of Picard rank 20 over $\mathbb{Q}$ up to $\mathbb{Q}$-isomorphism. Section 12 generalises Theorem 1 to K3 surfaces with Picard rank 20 over a quadratic extension of $\mathbb{Q}$. The paper concludes with the proof of the general case of Theorem 2 .

\section{Singular K3 surfaces}

The main invariant of a singular $\mathrm{K} 3$ surface $X$ is its transcendental lattice $T(X)$. Here we consider the Néron-Severi group $\mathrm{NS}(X)$ of divisors up to algebraic equivalence as a lattice in $H^{2}(X, \mathbb{Z})$ with cup-product. Then the transcendental lattice is the orthogonal complement

$$
T(X)=\mathrm{NS}(X)^{\perp} \subset H^{2}(X, \mathbb{Z}) .
$$

The following classification was first stated by Pjateckiı̌-Šapiro and Shafarevich [1971]. The proof was completed by Shioda and Inose [1977]: 
Theorem 3 [Piatetskiǔ-Shapiro and Shafarevich 1971; Shioda and Inose 1977]. The map $X \mapsto T(X)$ gives a bijection

$$
\{\text { Singular K3 surfaces }\}_{/ \cong} \stackrel{1: 1}{\longleftrightarrow}\left\{\begin{array}{c}
\text { positive-definite oriented } \\
\text { even lattices of rank two }
\end{array}\right\}_{/ \cong} .
$$

The injectivity of this map follows from the Torelli theorem for singular K3 surfaces [Piatetski1-Shapiro and Shafarevich 1971]. For the surjectivity, Shioda and Inose [1977] exhibited an explicit construction involving isogenous CM-elliptic curves $E, E^{\prime}$. This is often referred to as Shioda-Inose structure:

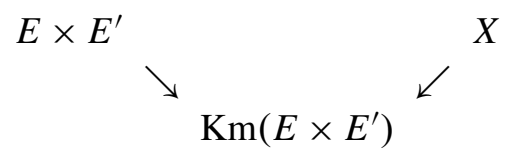

Here both rational maps are $2: 1$, and $T(X) \cong T\left(E \times E^{\prime}\right)$. Shioda and Inose exhibited the rational map $X \rightarrow \operatorname{Km}\left(E \times E^{\prime}\right)$ through base change of elliptic fibrations. Explicit equations were subsequently given by Inose [1978]. In [Schütt 2007b], Inose's results were improved to derive a model over the ring class field $H(d)$ associated to the discriminant $d=\operatorname{disc}(T(X))$ of the transcendental lattice (Lemma 33). Over some extension, one can moreover determine the $\zeta$-function of $X$ (Theorem 29).

The set of singular K3 surfaces over $\mathbb{Q}$ (up to $\mathbb{C}$-isomorphism) is finite by a result of Shafarevich [1996], quoted in Theorem 35. However, there is only one effective obstruction known for a singular K3 surface $X$ to be defined over $\mathbb{Q}$ : By [Schütt 2007b], the genus of $T(X)$ has to consist of a single class. (Shimada [2009] proved this first for the case of the fundamental discriminant $d$.) In other words, we require that its class group be only two-torsion:

$$
\mathrm{Cl}(T(X)) \cong(\mathbb{Z} / 2)^{g} .
$$

The general case will be treated in Section 13. There we will also provide a formulation in terms of fields of definition (Lemma 34).

The only drawback of relation (1) is that the class group $\mathrm{Cl}(T(X))$ does not recognise whether $T(X)$ is primitive. We know 101 discriminants $d<0$ such that the class group $\mathrm{Cl}(d)$ is only two-torsion. By a result of Weinberger [1973] there is at most one more such $d$, and in fact none under some condition on Siegel-Landau zeroes (which would follow from GRH). However, so far we lacked bounds for the degree of primitivity of $T(X)$. For Picard rank 20 over $\mathbb{Q}$, primitivity is part of Theorem 1 . For the general case, bounds for the degree of primitivity follow from Theorem 2 (see Section 13). 


\section{Modularity of singular K3 surfaces over $\mathbb{Q}$}

We shall now see that condition (1) can also be understood in terms of modularity. Here the modular motive is the compatible system of Galois representations attached to the transcendental lattice. Over some extension, this motive is related to Hecke characters by Theorem 29.

Throughout the paper, we fix the imaginary quadratic field $K=\mathbb{Q}(\sqrt{d})$ where $d<0$ is the discriminant of $X$. Write $d_{K}$ for the discriminant of $K$. Hence $d=$ $N^{2} d_{K}$.

Theorem 4 [Livné 1995]. Every singular K3 surface $X$ over $\mathbb{Q}$ is modular. The $L$-series of the transcendental lattice $T(X)$ is the Mellin transform of a Hecke eigenform of weight 3 with CM by $K$.

By a result of Ribet [1977], CM-newforms are associated to Hecke characters. Essentially, a Hecke character $\psi$ of $K$ is given by its conductor $\mathfrak{m}$, an ideal in the ring of integers $O_{K}$, and by its $\infty$-type $l$. Then $\psi$ satisfies

$$
\psi\left(\alpha \mathscr{O}_{K}\right)=\alpha^{l} \text { for all } \alpha \equiv 1 \bmod \mathfrak{m} .
$$

Let $\mathrm{N}_{K / \mathbb{Q}}$ denote the norm of $K / \mathbb{Q}$. The sum over all ideals $\mathfrak{a}$ of $\mathscr{O}_{K}$ that are relatively prime to $\mathfrak{m}$ gives the $L$-function of $\psi$ :

$$
L(\psi, s)=\sum_{\mathfrak{a}} \psi(\mathfrak{a}) \mathrm{N}_{K / \mathbb{Q}}(\mathfrak{a})^{s} .
$$

Through the inverse Mellin transform, $L(\psi, s)$ defines a newform of weight $k=$ $l+1$ and level $\left|\mathrm{N}_{K / \mathbb{Q}}(\mathfrak{m}) d_{K}\right|$. For the weight of the corresponding newform to be 3 , the Hecke character thus has to have $\infty$-type 2. Moreover, we require the newform to have Fourier coefficients in $\mathbb{Z}$. This is possible if and only if the class group of $K$ consists only of two-torsion (see Theorem 6). This condition is necessarily satisfied if (1) holds.

Example 5. Let $K$ be such that $\mathrm{Cl}(K) \cong(\mathbb{Z} / 2)^{g}$ with $d_{K} \neq-3,-4$. Then we can define a Hecke character $\psi$ of $K$ with trivial conductor and $\infty$-type 2 by setting

$$
\psi\left(\alpha \mathrm{O}_{K}\right)=\alpha^{2}
$$

for every principal ideal in $\mathrm{O}_{K}$ and choosing suitable values for a set of generators of $\mathrm{Cl}(K)$. Explicitly, throughout this paper let

$$
D= \begin{cases}-d_{K}, & \text { if } 4 \nmid d_{K}, \\ -d_{K} / 4, & \text { if } 4 \mid d_{K} .\end{cases}
$$

Assume that $p=\mathfrak{p} \overline{\mathfrak{p}}$ splits in $K$. Since $d_{K} \neq-3,-4$, we can write $p^{2}$ uniquely as

$$
p^{2}=x^{2}+D y^{2}, \quad x, y \in \frac{1}{2} \mathbb{N} \text {. }
$$


(Here $x, y \in \mathbb{N}$ unless $D=-d_{K}$.) Then $\psi(\mathfrak{p})= \pm(x \pm \sqrt{-D} y)$. For the corresponding newform $f=\sum a_{n} q^{n}$, we obtain

$$
a_{p}= \pm 2 x .
$$

Once a normalisation is fixed, $f$ has level $\left|d_{K}\right|$ and Fourier coefficients in $\mathbb{Z}$.

The newforms arising from different normalisations (that is, sign choices) are quadratic twists of each other. In general, consider a (quadratic) Dirichlet character $\chi$ and a newform $f=\sum a_{n} q^{n}$. Then we obtain the twisted Hecke eigenform

$$
f \otimes \chi=\sum_{n} a_{n} \chi(n) q^{n} .
$$

The classification in [Schütt 2009] says that the construction of Example 5 produces all Hecke characters and Hecke eigenforms with Fourier coefficients in $\mathbb{Z}$ after twisting:

Theorem 6 [Schütt 2009]. Let $K$ be an imaginary quadratic field. Then all Hecke characters of $K$ with fixed $\infty$-type $l$ such that the corresponding newform $f$ has coefficients in $\mathbb{Z}$, are identified under twisting. Moreover, there is such a Hecke character if and only if $\mathrm{Cl}(K) \subseteq(\mathbb{Z} / l)^{g}$ for some $g \in \mathbb{N}$.

Remark 7. If $d_{K} \neq-3,-4$, then we only have to consider quadratic twists. If $\chi$ is a quadratic Dirichlet character, then we twist the Hecke character by $\chi \circ \mathrm{N}_{\mathbb{Q}}^{K}$. In terms of the associated newform $f$, this corresponds to the quadratic twist in (2). For $d_{K}=-3,-4$, we also have to take cubic and biquadratic twisting into account. All these twists have geometric equivalents. For instance, any quadratic Dirichlet character can be identified with a Legendre symbol $\left(\frac{\delta}{.}\right)$ for some square-free $\delta \in \mathbb{Z}$. Then consider an elliptic curve (or a general equation of this type)

$$
E: y^{2}=g(x) \quad \text { and twist } \quad E_{\delta}: \delta y^{2}=g(x) .
$$

For geometric equivalents of cubic and biquadratic twists, see Remark 27.

\section{K3 surfaces of Picard rank 20 over $\mathbb{Q}$ : Examples}

In this section, we recall two of the most elementary examples of K3 surfaces of Picard rank 20 over $\mathbb{Q}$. Both use elliptic fibrations with section. For further examples, the reader is referred to Section 10.

Example 8. There is a unique complex elliptic K3 surface $X$ with a fibre of type $I_{19}$. The fibration can be defined over $\mathbb{Q}$. This follows from work of Hall [1971] and was studied in detail by Shioda [2003]. A simple explicit Weierstrass equation is derived in [Schütt and Schweizer 2007]:

$$
X: y^{2}=x^{3}+\left(t^{4}+t^{3}+3 t^{2}+1\right) x^{2}+2\left(t^{3}+t^{2}+2 t\right) x+t^{2}+t+1 .
$$


Let $U$ denote the hyperbolic plane generated by a general fibre and the zerosection. It is immediate that the Néron-Severi lattice of $X$ (over $\overline{\mathbb{Q}}$ ) can be written as

$$
\mathrm{NS}(X)=U \oplus A_{18}(-1)=\left(\begin{array}{ll}
0 & 1 \\
1 & 0
\end{array}\right) \oplus A_{18}(-1) .
$$

In particular, $X$ is a singular K3 surface. The Picard rank of $X$ over $\mathbb{Q}$ is 20 if and only if the all components of the special fibre are defined over $\mathbb{Q}$ (that is, if the special fibre has split multiplicative reduction). This can be achieved by an appropriate twist as in (3), and was first exhibited in [Schütt and Top 2006]. The model in (4) has the fibre of type $I_{19}$ at $t=\infty$. The fibre is split multiplicative, so the Picard rank of the surface over $\mathbb{Q}$ is already 20. The corresponding Hecke eigenform has level 19 by [Schütt and Top 2006] (see [Schütt 2009, Table 1]).

The next example goes back to [Tate 1974]. It has been studied very concretely in [Hulek and Verrill 2005].

Example 9. Let $X$ denote the universal elliptic curve for $\Gamma_{1}(7)$. Since this group has genus 0 , the base curve is $\mathbb{P}^{1}$. One the other hand, the space of cusp forms $S_{3}\left(\Gamma_{1}(7)\right)$ is one-dimensional, so $X$ has geometric genus $p_{g}(X)=1$. It follows that $X$ is a $\mathrm{K} 3$ surface. By general theory, the elliptic surface $X$ has a model over $\mathbb{Q}$ with a section $P$ of order 7 also defined over $\mathbb{Q}$. Such a model was first given by Tate [1974]:

$$
X: y^{2}+\left(1+t-t^{2}\right) x y+\left(t^{2}-t^{3}\right) y=x^{3}+\left(t^{2}-t^{3}\right) x^{2} .
$$

Here $P=(0,0)$ is a point of order 7 . In the following, we shall employ an abstract approach to show that $X$ has Picard rank 20 over $\mathbb{Q}$.

The quotient of $X$ by translation by $P$ gives rise to another elliptic K3 surface after resolving singularities. Hence the configuration of singular fibres can only be $[1,1,1,7,7,7]$. In particular, $X$ is a singular K3 surface. We claim that the above model has Picard rank 20 over $\mathbb{Q}$. Equivalently, each reducible fibre is completely defined over $\mathbb{Q}$. To prove this, we show that $P$ meets each $I_{7}$ fibre in a different nontrivial component.

We employ Shioda's theory [1990] of Mordell-Weil lattices and the height pairing. As a torsion section, $P$ has height 0 . Since $P$ does not meet the 0 -section, we can compute the height directly as

$$
h(P)=4-\text { (correction terms for reducible fibres). }
$$

Here the correction terms are $(n(7-n)) / 7$ according to the component $\Theta_{n}$ which $P$ meets (cyclically numbered so that the zero-section meets $\Theta_{0}$ ). The only way to obtain $h(P)=0$ is

$$
0=h(P)=4-\frac{6}{7}-\frac{10}{7}-\frac{12}{7} .
$$


Since $P$ intersects each $I_{7}$ fibre at a nontrivial component, these special fibres are split multiplicative. Moreover, as the components differ for each $I_{7}$ fibre, their cusps cannot be conjugate. Hence all fibre components are defined over $\mathbb{Q}$, and the claim follows.

Remark 10. The same argument applies to other modular elliptic K3 surfaces, but not to all of them. For instance, the universal elliptic curve for $\Gamma(4)$ is a Kummer surface. Hence it cannot have Picard rank 20 over $\mathbb{Q}$ by the next remark. This argument will also be used in the proof of the primitivity of the transcendental lattice (Lemma 22). Alternatively, we could also argue with the Weil pairing. Since the Weil pairing has image $\mu_{4}$, the fourth roots of unity, we deduce that $M W(X / \mathbb{Q}) \subset \mathbb{Z} / 4 \times \mathbb{Z} / 2$. Then we apply the inverse argument of Example 9 to a 4-torsion section which is not defined over $\mathbb{Q}$. This implies that there are singular fibres which are not completely defined over $\mathbb{Q}$.

Remark 11 (Singular abelian surfaces). The situation for abelian surfaces is different: Let $A$ be a singular complex abelian surface, that is, $\rho(A)=4$. Then $A \cong E \times E^{\prime}$ for isogenous CM-elliptic curves $E, E^{\prime}$ by a result of Shioda and Mitani [1974]. However, as Shioda [2005] noted, Picard rank 4 over $\mathbb{Q}$ is impossible. This is a consequence of the cohomology structure of abelian varieties and carries over to Kummer surfaces (see also Remark 10 and Lemma 22).

\section{The Artin-Tate conjecture}

Let $X$ be a K3 surface of Picard rank 20 over $\mathbb{Q}$. In order to prove Theorem 1, we will consider the reductions of $X$ at the good primes $p$ that split in $K$ and apply the Artin-Tate conjecture.

Let $p$ be a prime of good reduction of $X$. Then the reduction morphism induces embeddings

$$
\mathrm{NS}(X / \mathbb{Q}) \hookrightarrow \mathrm{NS}\left(X / \mathbb{F}_{p}\right) \quad \text { and } \quad \mathrm{NS}(X / \overline{\mathbb{Q}}) \hookrightarrow \mathrm{NS}\left(X / \overline{\mathbb{F}}_{p}\right),
$$

which are isometries onto the image. For almost all $p$, these embeddings are primitive. This follows from Shimada's argumentation [2009, §2.2], since the proof for the case of supersingular reduction can be generalised directly. For the remainder of the paper, we will only consider good primes where the reduction is good and the embeddings in (5) are primitive.

On $X / \mathbb{F}_{p}$ we have the Frobenius endomorphism Frob ${ }_{p}$ raising coordinates to their $p$ th powers. We want to consider the induced action on cohomology. For this, we fix a prime $\ell \neq p$ and work with étale $\ell$-adic cohomology of the base change $\bar{X}=X_{\overline{\mathbb{F}}_{p}}$ to an algebraic closure $\overline{\mathbb{F}}_{p}$ of $\mathbb{F}_{p}$. Then we consider the induced 
map $\operatorname{Frob}_{p}^{*}$ on $H_{\mathrm{et}}^{2}\left(\bar{X}, \mathbb{Q}_{\ell}\right)$ and its reciprocal characteristic polynomial

$$
P\left(X / \mathbb{F}_{p}, T\right)=\operatorname{det}\left(1-\operatorname{Frob}_{p}^{*} T ; H_{\text {êt }}^{2}\left(\bar{X}, \mathbb{Q}_{\ell}\right)\right) .
$$

Frob $_{p}^{*}$ acts through a permutation on the algebraic cycles in $H_{\mathrm{ett}}^{2}\left(\bar{X}, \mathbb{Q}_{\ell}\right)$. More precisely, it operates as multiplication by $p$ on $\mathrm{NS}\left(X / \mathbb{F}_{p}\right)$ and in particular on the image of $\mathrm{NS}(X / \mathbb{Q})$ under the primitive embedding (5). In the present case, $X$ has Picard rank 20 over $\mathbb{Q}$ and discriminant $d$. Let $f=\sum a_{n} q^{n}$ denote the associated newform by Theorem 4 . Then

$$
P\left(X / \mathbb{F}_{p}, T\right)=(1-p T)^{20}\left(1-a_{p} T+\left(\frac{d}{p}\right) p^{2} T^{2}\right) .
$$

The Tate conjecture [1965] relates the shape of the zeroes of $P\left(X / \mathbb{F}_{p}, T\right)$ to the Picard number: Conjecturally for any smooth projective surface $X$ over $\mathbb{F}_{p}$, it predicts

$$
\begin{aligned}
& \rho\left(X / \mathbb{F}_{p}\right)=\#\left\{\text { zeroes } T=\frac{1}{p} \text { of } P\left(X / \mathbb{F}_{p}, T\right)\right\}, \\
& \rho\left(X / \overline{\mathbb{F}}_{p}\right)=\#\left\{\text { zeroes } T=\zeta \frac{1}{p} \text { of } P\left(X / \mathbb{F}_{p}, T\right) \text { where } \zeta \text { is a root of unity }\right\} .
\end{aligned}
$$

Here we count the zeroes with multiplicities. Since Frob $_{p}$ acts as multiplication by $p$ on $N S\left(X / \mathbb{F}_{p}\right)$, we always have $\leq$ in the above equations. For instance, the Tate conjecture is known for elliptic K3 surfaces [Artin and Swinnerton-Dyer 1973]. By [Milne 1975] (see the addendum cited for characteristic two), it is equivalent to the Artin-Tate conjecture:

Conjecture 12 (Artin and Tate [Tate 1966]). Let $X / \mathbb{F}_{p}$ be a smooth projective surface. Let $\alpha(X)=\chi(X)-1+\operatorname{dim} \operatorname{Pic} \operatorname{Var}(X)$. Then

$$
\left.\frac{P\left(X / \mathbb{F}_{p}, T\right)}{(1-p T)^{\rho\left(X / \mathbb{F}_{p}\right)}}\right|_{T=\frac{1}{p}}=\frac{\left|\operatorname{Br}\left(X / \mathbb{F}_{p}\right)\right|\left|\operatorname{discr}\left(\mathrm{NS}\left(X / \mathbb{F}_{p}\right)\right)\right|}{p^{\alpha(X)}\left|\mathrm{NS}\left(X / \mathbb{F}_{p}\right)_{\text {tor }}\right|^{2}}
$$

Remark 13. By [Liu et al. 2005], $\left|\operatorname{Br}\left(X / \mathbb{F}_{p}\right)\right|$ is always a square. For $\mathrm{K} 3$ surfaces, $\alpha(X)=1$ and the Néron-Severi group is torsion-free, since numerical and algebraic equivalence coincide. Hence (7) simplifies to

$$
\left.\frac{P\left(X / \mathbb{F}_{p}, T\right)}{(1-p T)^{\rho\left(X / \mathbb{F}_{p}\right)}}\right|_{T=\frac{1}{p}}=\frac{1}{p}\left|\operatorname{Br}\left(X / \mathbb{F}_{p}\right)\right|\left|\operatorname{discr}\left(\operatorname{NS}\left(X / \mathbb{F}_{p}\right)\right)\right| .
$$

We shall now specialise to the situation where $X$ is a K3 surface with Picard rank 20 over $\mathbb{Q}$ and $p$ is a good split prime. The Fourier coefficient $a_{p}$ can be computed in terms of Example 5. In particular, it is never a multiple of $p$. Hence the zero $T=(1 / p)$ of $P\left(X / \mathbb{F}_{p}, T\right)$ has multiplicity exactly 20 , and there is no further zero $T=\zeta(1 / p)$. It follows that $\rho\left(X / \mathbb{F}_{p}\right)=\rho\left(X / \overline{\mathbb{F}}_{p}\right)=20$. In particular, 
the Tate conjecture holds for $X$ over $\mathbb{F}_{p}$. From (5) we deduce

$$
\mathrm{NS}(X / \overline{\mathbb{Q}})=\mathrm{NS}(X / \mathbb{Q})=\mathrm{NS}\left(X / \mathbb{F}_{p}\right)=\mathrm{NS}\left(X / \overline{\mathbb{F}}_{p}\right)
$$

and thus

$$
\operatorname{discr}(\mathrm{NS}(X / \mathbb{Q}))=\operatorname{discr}\left(\mathrm{NS}\left(X / \mathbb{F}_{p}\right)\right)=d=N^{2} d_{K} .
$$

Hence the Artin-Tate conjecture for $X / \mathbb{F}_{p}(8)$ gives, with $M^{2}=\left|\operatorname{Br}\left(X / \mathbb{F}_{p}\right)\right|$,

$$
2 p-a_{p}=M^{2}|d|=(M N)^{2}\left|d_{K}\right| \text {. }
$$

The proof of Theorem 1 now proceeds in three steps:

(A) The imaginary quadratic field $K$ has class number one (Corollary 15).

(B) The discriminant $d$ has class number one (Corollary 20).

(C) The transcendental lattice $T(X)$ is primitive (Lemma 22).

As a by-product, we will also determine the possible shapes of the associated newform $f$ (Lemma 17).

\section{Class number of $K$}

In this section, we will prove that $K$ has class number one. We achieve this through the following proposition:

Proposition 14. Let $p$ split in $K$ and let $a_{p} \in \mathbb{Z}$ be the coefficient of a newform of weight 3 with $C M$ by $K$. Then (9) implies that $p$ splits into principal ideals in $K$.

Proof. By Example 5, we can write $a_{p}=2 z$ with $z= \pm x \in \frac{1}{2} \mathbb{Z}$. By (9), we have

$$
p-z=\frac{m^{2} D}{2}
$$

for some $m \in \mathbb{N}$. On the other hand, $p^{2}=z^{2}+D y^{2}$ for some $y \in \frac{1}{2} \mathbb{N}$ by assumption, that is,

$$
p^{2}-z^{2}=D y^{2}
$$

Dividing (11) by (10), we obtain

$$
p+z=2\left(\frac{y}{m}\right)^{2} .
$$

Now we add (10) and (12) and divide by two to derive

$$
p=\left(\frac{y}{m}\right)^{2}+D\left(\frac{m}{2}\right)^{2}
$$

Since $m / 2 \in \frac{1}{2} \mathbb{N}$, the same holds for $y / m$. We deduce that $p$ splits into principal ideals in $K$. 
Corollary 15. Let $X$ be a $K 3$ surface of Picard rank 20 over $\mathbb{Q}$. Then its $C M$-field $K$ has class number one.

Proof. By the Artin-Tate conjecture, Equation (9) holds at all but finitely many $p$ that split in $K$. By Proposition 14, each of these $p$ splits into principal ideals in $K$. Hence $K$ has class number one.

\section{Shape of $f$}

If $K$ has class number one, we can describe the CM-newforms of $K$ even more explicitly in terms of Example 5. Here we only have to take extra care of the special cases $d_{K}=-3,-4$ where $0_{K} \neq\{ \pm 1\}$. For this purpose, let

$$
D^{\prime}= \begin{cases}27, & \text { if } d_{K}=-3, \\ 4, & \text { if } d_{K}=-4, \\ D, & \text { if } d_{K} \neq-3,-4\end{cases}
$$

Example 16 (Class number one). Let $K$ have class number one. Let $D^{\prime}$ as above. If $p$ splits in $K$, then we rewrite (13) uniquely as

$$
p=x^{2}+D^{\prime} y^{2}, \quad x, y \in \frac{1}{2} \mathbb{N} .
$$

The corresponding Hecke character $\psi$ of $\infty$-type 2 sends the prime ideal $(x+$ $\sqrt{-D^{\prime}} y$ ) to its square. We obtain the newform $f_{K}$ of weight 3 and level $D^{\prime}$ from [Schütt 2009, Table 1] with coefficients

$$
a_{p}=2\left(x^{2}-D^{\prime} y^{2}\right) .
$$

Lemma 17. Let $X$ be a $K 3$ surface of Picard rank 20 over $\mathbb{Q}$. Let $f$ denote the associated newform.

(i) If $d_{K} \neq-3,-4$, then $f=f_{K}$.

(ii) If $d_{K}=-4$, then $f$ is a quadratic twist of $f_{K}$.

(iii) If $d_{K}=-3$, then $f$ is a cubic twist of $f_{K}$.

Proof. Assume that $d_{K} \neq-3,-4$. Let $p$ be a split prime as in Example 16. By Theorem 6, $f$ has the coefficient

$$
a_{p}= \pm 2\left(x^{2}-D y^{2}\right) .
$$

Inserting into (9) gives

$$
2\left(x^{2}+D y^{2} \mp\left(x^{2}-D y^{2}\right)\right)=m^{2} D .
$$

Since $d_{K}$ is not a square and neither is $D$, it follows that only the minus sign in (16) is possible. That is, in (15), only the plus sign occurs. By definition $f=f_{K}$. 
If $d_{K}=-4$ and $p=x^{2}+4 y^{2}$, then

$$
a_{p}=\left\{\begin{array}{l} 
\pm 2\left(x^{2}-4 y^{2}\right), \\
\pm 8 x y .
\end{array}\right.
$$

The second case occurs (at some split $p$ ) if and only if $f$ is a biquadratic twist of $f_{K}$. Only the first case is compatible with (9), since in the second case

$$
2 p-a_{p}=2\left(x^{2}+4 y^{2} \mp 4 x y\right)=2(x \mp 2 y)^{2} \neq 4 n^{2} .
$$

Hence $f$ is a quadratic twist of $f_{K}$.

A similar argument rules out quadratic and sextic twists of $f_{K}$ for $d_{K}=-3$ : Here we can always write the coefficients of $f$ as

$$
a_{p}= \pm 2\left(x^{2}-3 y^{2}\right) \quad \text { where nonuniquely } \quad p=x^{2}+3 y^{2}, \quad x, y \in \frac{1}{2} \mathbb{N} .
$$

By the argument of case (i), only the plus sign occurs. This implies that $f$ is a cubic twist of $f_{K}$.

\section{Class number of $d$}

Let $X$ be a K3 surface of Picard rank 20 over $\mathbb{Q}$. Denote the associated newform by $f=\sum a_{n} q^{n}$. We can rephrase Lemma 17 and its proof as follows: At every good split prime $p$, we can write (nonuniquely if $D \neq D^{\prime}$ )

$$
p=x_{p}^{2}+D y_{p}^{2} \quad \text { such that } \quad a_{p}=2\left(x_{p}^{2}-D y_{p}^{2}\right) \quad \text { and } \quad 4 D y_{p}^{2}=M_{p}^{2}|d| .
$$

By construction, we have either $d_{K}=-4 D$ and $y \in \mathbb{N}$ or $d_{K}=-D$ and $y \in \frac{1}{2} \mathbb{N}$. Recall that $d=N^{2} d_{K}$ and $d_{K}$ has class number one by Corollary 15 . We want to find all $d$ which are compatible with Picard rank 20 over $\mathbb{Q}$. In other words, we search for all $N \mid M_{p}$ which are simultaneously possible in (17) at all good split $p$.

Observation 18. Let gcd denote the greatest common divisor in $\mathbb{N}$ if $d_{K}=-4 D$, or in $\frac{1}{2} \mathbb{N}$ if $d_{K}=-D$. Let $y_{p}$ be given by (17) at a good split prime $p$. Then

$$
N \mid \operatorname{gcd}\left(y_{p} ; p \operatorname{good} \text { split prime for } X\right) \text {. }
$$

Hence, if for instance there was a $y_{p}=1$ ( or $y_{p}=\frac{1}{2}$ in the case $d_{K}=-D$ ) occurring, then $d=d_{K}$ (and $N=M_{p}=1$ ) would follow. However, this need not be the case in general. To see this, let the associated newform $f$ have level 27 . Then by construction $3 \mid y_{p}$ for all split $p$. Hence at least $d=-3$ and $d=-27$ would be possible a priori.

To bound $d$ (or $N$ ) in general, we need information on the greatest common divisor of the $y_{p}$. This divisibility problem translates into class group theory through representations of primes by quadratic forms: 
Lemma 19. Let $d<0$ and let $Q=\left(\begin{array}{ll}2 & b \\ b & 2 c\end{array}\right)$ be a quadratic form of discriminant $d$. For $r \in \mathbb{N}$, the following are equivalent:

(i) For almost every prime $p$ represented by $Q$, there is a representation

$$
p=u^{2}+b u v+c v^{2}, \quad u, v \in \mathbb{Z},
$$

such that $r \mid v$.

(ii) $h(d)=h\left(d r^{2}\right)$.

Proof. Note that $Q$ always represents the principal class in $\mathrm{Cl}(d)$. Hence, if $h(d)=$ $h\left(d r^{2}\right)$, the quadratic form

$$
Q_{r}=\left(\begin{array}{cc}
2 & b r \\
b r & 2 c r^{2}
\end{array}\right)
$$

in $\mathrm{Cl}\left(d r^{2}\right)$ represents the same primes as $Q$ (the principal ones). Thus $r \mid v$ for all these $p$.

Conversely, assume that $r \mid v$ for almost all $p$ represented by $Q$. Thus all these $p$ are represented by $Q_{r}$ as well. Since the split primes are equally distributed on the classes that represent them, we obtain $h(d) \geq h\left(d r^{2}\right)$. On the other hand, $h(d) \leq h\left(r^{2} d\right)$ holds trivially. Hence the class numbers $h(d)$ and $h\left(d r^{2}\right)$ have to coincide.

Corollary 20. Let $X$ be a $K 3$ surface of Picard rank 20 over $\mathbb{Q}$. Then the transcendental lattice has discriminant d of class number one.

Proof. By Corollary $15, d_{K}$ has class number one. Assume that $d \neq d_{K}$, that is, there is some $r$ dividing all $y_{p}$ in (17). To apply Lemma 19, we have to relate divisibility of $y_{p}$ and $v_{p}$. We consider the following quadratic forms:

$$
\left(Q=\begin{array}{cc}
2 & 0 \\
0 & 2 D
\end{array}\right) \text { if } d_{K} \text { is even, } \quad Q=\left(\begin{array}{cc}
2 & 1 \\
1 & (D+1) / 2
\end{array}\right) \text { if } d_{K} \text { is odd. }
$$

If $d_{K}$ is even, then $d_{K}=-4 D$ and $v_{p}=y_{p} \in \mathbb{N}$. Hence $h(d)=h\left(d_{K}\right)=1$ follows from Lemma 19. If $d_{K}$ is odd, then we can rewrite (18) in half-integers:

$$
p=u_{p}^{2}+u_{p} v_{p}+\frac{D+1}{4} v_{p}^{2}=\left(u_{p}+\frac{v_{p}}{2}\right)^{2}+D\left(\frac{v_{p}}{2}\right)^{2} .
$$

Hence, divisibility of $y_{p}$ in $\frac{1}{2} \mathbb{N}$ translates into divisibility of $v_{p} \in \mathbb{N}$ and vice versa. Again we deduce $h(d)=h\left(d_{K}\right)=1$ by Lemma 19 .

Remark 21. Let $X$ be a K3 surface of Picard rank 20 over $\mathbb{Q}$. If $d \neq d_{K}$, it is immediate from the above argument that the associated newform $f$ has a particular shape. For $d=-28$, this newform is uniquely determined with level 7 by Lemma 17. In the other three cases $(d=-12,-16,-27)$, it is easily checked that the condition $r \mid y_{p}$ fixes a unique Hecke character. We find that $f$ is the unique newform of weight 3 and level $|d|$. 


\section{Primitivity of $T(X)$}

We have seen that a K3 surface with Picard rank 20 over $\mathbb{Q}$ has discriminant of class number one. Hence there are a priori 17 possibilities for the transcendental lattice:

- the 13 primitive lattices of class number one, corresponding to isomorphism classes of CM-elliptic curves over $\mathbb{Q}$ through the Shioda-Inose structure, and

- the 4 imprimitive lattices of discriminant $d=-12,-16,-27,-28$.

In this section, we will rule out the second case.

Lemma 22. Let $X$ be a $K 3$ surface of Picard rank 20 over $\mathbb{Q}$. Then $T(X)$ is primitive.

Proof. Assume that $T(X)$ is not primitive. By Corollary 20, we are in the second case above. We shall treat even and odd discriminants separately.

If $d$ is even in the second case above, then the transcendental lattice $T(X)$ has intersection form $2 Q$ for $Q \in \mathrm{Cl}\left(d^{\prime}\right)$ where $4 d^{\prime}=d$. It follows from [Shioda and Inose 1977] that $X$ is the Kummer surface of an abelian surface $A$ such that the transcendental lattice $T(A)$ has intersection form $Q$. By Remark $11, \rho(A / \mathbb{Q})<4$ and $\rho(X / \mathbb{Q}) \leq \rho(A / \mathbb{Q})+16<20$.

If $d$ is odd, that is, $d=-27$, we consider Inose's fibration on $X$ [Inose 1978; Shioda 2006]. In the present case, $K=\mathbb{Q}(\sqrt{-3})$, and $X$ arises from the ShiodaInose construction (see Section 2) for the following elliptic curves:

$$
E \text { with } \mathrm{CM} \text { by } \mathrm{O}_{K} \quad \text { and } \quad E^{\prime} \text { with } \mathrm{CM} \text { by } \mathbb{Z}+30_{K} \text {. }
$$

In particular, $j(E)=0$. It follows from [Inose 1978] that $X$ admits the isotrivial elliptic fibration

$$
X: \quad y^{2}=x^{3}+t^{5}\left(3 t^{2}-2 \cdot 11 \cdot 23 t+3\right) .
$$

Here the singular fibres have type $I I^{*}, I I^{*}, I I, I I$, and the Mordell-Weil group over $\overline{\mathbb{Q}}$ has rank two. The generic fibre has $\mathrm{CM}$ by $\mathrm{O}_{K}$. Let $\omega$ denote a primitive third root of unity acting on $X$ via $x \mapsto \omega x$. If $P$ is a section of the elliptic surface, then so is $\omega^{*} P$. Since the singular fibres admit no nontrivial torsion sections, these sections are independent. Since this argumentation applies to any twist $Y$ of $X$, $\operatorname{Gal}(\mathbb{Q}(\sqrt{-3}) / \mathbb{Q})$ always acts nontrivially on $M W(Y)$. Hence rk $M W(Y / \mathbb{Q})<2$ and in particular $\rho(Y / \mathbb{Q})<20$.

\section{Existence of K3 surfaces of Picard rank 20 over $\mathbb{Q}$}

There are 13 primitive lattices $T$ of class number one appearing in Theorem 1. For each of them one can ask whether there is a K3 surface with Picard rank 20 over $\mathbb{Q}$ and this transcendental lattice. Elkies [2007] announced in that this holds true 
for each $T$. It follows that for each of these surfaces, one such model is given by Inose's fibration for the CM-elliptic curve corresponding to $T$, as exhibited over $\mathbb{Q}$ in [Schütt 2007b].

However, for Inose's fibration, the nontrivial sections are often not immediate. In the cases at hand, there are two fibres of type $I I^{*}$ plus an additional reducible fibre of type $I_{2}$. Hence the Mordell-Weil rank is one. Elkies recently computed the Mordell-Weil generator of height $|d| / 2$ explicitly for all these fibrations [Elkies 2008a].

For the reader's convenience we include a list of different models of these K3 surfaces where the Picard rank 20 over $\mathbb{Q}$ becomes evident. These models are given in terms of elliptic fibrations with configuration of singular fibres and the abstract structure of the Mordell-Weil group. We also include a reference, but naturally the given models are far from unique. Other models may be found in [Elkies 2008b; Schütt 2007a; Top and Yui 2007] for instance. Explanations follow the table.

\begin{tabular}{|r|c|c|l|}
\hline$d$ & configuration & $M W$ & reference \\
\hline-3 & {$\left[1^{3}, 3,12^{*}\right]$} & $\mathbb{Z} / 4$ & Lemma 26 \\
-4 & {$\left[0^{*}, I I I^{*}, I I I^{*}\right]$} & $\mathbb{Z} / 2$ & Lemma 25 \\
-7 & {$\left[1^{3}, 7^{3}\right]$} & $\mathbb{Z} / 7$ & Example 9 \\
-8 & {$\left[1,4, I I I^{*}, I I^{*}\right]$} & $\{0\}$ & {$[$ Schütt 2007a, §7] } \\
-11 & {$\left[1^{3}, 11, I I^{*}\right]$} & $\{0\}$ & {$[$ Schütt 2006, (III.2)] } \\
-12 & {$\left[2,3, I I I^{*}, I I^{*}\right]$} & $\{0\}$ & {$[$ Schütt 2007a, §7] } \\
-16 & {$\left[2,8,1^{*}, 1^{*}\right]$} & $\mathbb{Z} / 4$ & {$[$ Schütt 2007a, §7] } \\
-19 & {$\left[1^{5}, 19\right]$} & $\{0\}$ & Example 8 \\
-27 & {$\left[1^{4}, 2,9^{2}\right]$} & $\mathbb{Z}+\mathbb{Z} / 3$ & Example 23 \\
-28 & {$\left[1^{6}, 6,12\right]$} & $\mathbb{Z}^{2}$ & {$[$ Elkies 2008b, §5] } \\
-43 & {$\left[1^{6}, 6,12\right]$} & $\mathbb{Z}^{2}$ & {$[$ Elkies 2008b, §5] } \\
-67 & {$\left[1^{3}, 4,7, I I^{*}\right]$} & $\mathbb{Z}$ & {$[$ Elkies 2008b, §4] } \\
-163 & {$\left[1^{6}, 6,12\right]$} & $\mathbb{Z}^{2}$ & {$[$ Elkies 2008b, §5] } \\
\hline
\end{tabular}

For $\boldsymbol{d}=\mathbf{- 8 , - 1 2}$, it was shown in [Schütt 2007a, §7] that the named fibrations are defined over $\mathbb{Q}$. To obtain Picard rank 20 over $\mathbb{Q}$, it suffices to apply a quadratic twist as in Example 8 such that the fibre of type $I_{4}$ or $I_{3}$, respectively, becomes split-multiplicative.

For $\boldsymbol{d}=\mathbf{- 1 1}$, the following Weierstrass form was derived in [Schütt 2006]:

$$
y^{2}=x^{3}+t^{2}\left(t^{2}+3 t+1\right) x^{2}+t^{4}(2 t+4) x+t^{5}(t+1) .
$$

This fibration has a $I I^{*}$ fibre at 0 and a split-multiplicative fibre of type $I_{11}$ at $\infty$. 
For $\boldsymbol{d}=\mathbf{- 1 6}$, we realise the surface as a quadratic base change of the extremal rational elliptic surface with configuration $\left[1,4,1^{*}\right]$. It has a rational 4-torsion section $P$ which meets the singular fibres $I_{4}$ at a near and $I_{1}^{*}$ at a far component [Miranda and Persson 1986]. This implies that all fibre components are defined over $\mathbb{Q}$. The same argumentation applies to the base changed surface. Here we choose the base change in such a way that the $I_{1}^{*}$ fibres sit above rational cusps.

Example 23 (Discriminant $\boldsymbol{d}=\mathbf{- 2 7}$ ). For this discriminant, we searched the onedimensional family of elliptic K3 surfaces with the given configuration $\left[1^{4}, 2,9^{2}\right]$ and a 3-torsion section for an appropriate specialisation. Using techniques from [Elkies and Schütt 2008], we found

$$
X: y^{2}+3\left(2 t^{2}+1\right) x y+\left(1-t^{2}\right)^{3} y=x^{3} .
$$

This elliptic surface has 3-torsion sections with zero $x$-coordinate and an independent section $P$ over $\mathbb{Q}$ with $x$-coordinate $x(P)=(t-1)^{3}$ and height $h(P)=3 / 2$. The $I_{9}$ fibres are located at $t= \pm 1$ and split-multiplicative. Hence $X$ has Picard rank 20 over $\mathbb{Q}$. Using the height pairing [Shioda 1990], one can show that neither $P$ nor its translates by the torsion sections are 3-divisible. Hence $X$ has discriminant

$$
d=-h(P) \frac{\operatorname{disc}\left(A_{1}\right) \operatorname{disc}\left(A_{8}\right)^{2}}{|M W(X)|^{2}}=-27 .
$$

\section{Classification up to $\mathbb{Q}$-isomorphism}

So far, we have only considered K3 surfaces up to isomorphism over $\mathbb{C}$. Then a singular K3 surface $X$ is identified by its transcendental lattice $T(X)$ (Theorem 3 ). In this section, we answer the question which $\mathbb{Q}$-isomorphism classes have Picard rank 20 over $\mathbb{Q}$. This is closely related to the precise shape of the corresponding Hecke eigenform (compare Lemma 17).

We will work with Inose's elliptic fibration. In this context, one should always have quadratic twisting as in (3) in mind. This operation twists the modular forms. Notably it also twists sections and affects singular fibres of types $I V, I V^{*}, I_{m}^{*}, I_{n}(n>2)$. Our first result concerns the case $d \neq-3,-4$ :

Proposition 24. Let $0>d \neq-3,-4$ of class number one. Up to $\mathbb{Q}$-isomorphism, there is a unique $K 3$ surface $X$ of discriminant $d$ and Picard rank 20 over $\mathbb{Q}$.

Proof. The existence was shown in the previous section. We work with Inose's fibration with reducible singular fibres $I_{2}, I I^{*}, I I^{*}$ and a section $P$ of height $h(P)=$ $|d| / 2$. Over $\mathbb{C}$, such a fibration is unique [Shioda 2006]. Over $\mathbb{Q}$, this only leaves quadratic twists (for $d \neq-3,-4$ ). But then the condition that the section $P$ be defined over $\mathbb{Q}$ distinguishes the unique twist with Picard rank 20 over $\mathbb{Q}$. 
Lemma 25. Let $d=-4$. Consider the extremal elliptic $K 3$ surface

$$
X: y^{2}=x^{3}-t^{3}(t-1)^{2} x,
$$

with singular fibres $I I I^{*}$ at 0 and $\infty$ and $I_{0}^{*}$ at 1 and two-torsion section $(0,0)$. Then any $K 3$ surface with discriminant $d$ and Picard rank 20 over $\mathbb{Q}$ is $\mathbb{Q}$-isomorphic to a quadratic twist of $X$.

Proof. The configuration determines a unique elliptic fibration over $\mathbb{C}$. Over $\mathbb{Q}$, we distinguish biquadratic twists

$$
X_{\delta}: \quad y^{2}=x^{3}-\delta t^{3}(t-1)^{2} x, \quad \delta \in \mathbb{Q}^{*} .
$$

All fibre components are defined over $\mathbb{Q}$ with the possible exception of the simple components of the $I_{0}^{*}$ fibre which do not meet the zero section. These components are endowed with the Galois action of the extension $\mathbb{Q}\left(x^{3}-\delta x\right) / \mathbb{Q}$. Hence all components are defined over $\mathbb{Q}$ if and only if $\delta$ is a square in $\mathbb{Q}^{*}$. This corresponds to the quadratic twist of (19) by $\sqrt{\delta}$ as in (3).

Lemma 26. Let $d=-3$. Consider Inose's fibration

$$
X: y^{2}=x^{3}-t^{5}(t+1)^{2}
$$

with singular fibres $I I^{*}$ at 0 and $\infty$ and $I V$ at -1 . Then any $K 3$ surface with discriminant $d$ and Picard rank 20 over $\mathbb{Q}$ is $\mathbb{Q}$-isomorphic to a cubic twist of $X$.

Different elliptic fibrations on this surface have been studied in [Schütt 2008]. We omit the proof, which is analogous to the previous one.

Remark 27. If $d=-3$ or -4 , then there are infinitely many possible associated newforms by Lemma 17. By the previous two lemmata, each of these twists (quadratic and cubic) is associated to a unique K3 surface of Picard rank 20 over $\mathbb{Q}$.

\section{K3 surfaces with Picard rank 20 over a quadratic extension}

In the next section, we will apply our methods to fields of definition of general singular K3 surfaces and their Néron-Severi lattices. To give a flavor of the ideas involved, we first give a full treatment of K3 surfaces with Picard rank 20 over a quadratic extension of $\mathbb{Q}$. We keep the techniques and notation above.

Proposition 28. Let $L$ be a quadratic extension of $\mathbb{Q}$ and $X$ be a $K 3$ surface with Picard rank 20 over $L$. As before, let $T(X)$ denote the transcendental lattice, $d$ its discriminant and $K=\mathbb{Q}(\sqrt{d})$.

(i) If $L=K$, then $d$ has class number one.

(ii) If $L \neq K$, then $d$ has class number one or two. In the latter case, the compositum $L K$ agrees with the ring class field $H(d)$. 
Proof. We consider all those primes $p$ that split in both $K$ and $L$. Let $\mathfrak{p} \mid p$ in $L$. Then $\mathbb{F}_{\mathfrak{p}}=\mathbb{F}_{p}$ and again $\rho\left(X / \mathbb{F}_{p}\right)=20$. As before we will apply the Artin-Tate conjecture to the reduction of $X$ at $\mathfrak{p}$. For this, we need the coefficient $a_{\mathfrak{p}}$ of the characteristic polynomial of Frob $\mathfrak{p}$ as in (6). Even if $X$ is not defined over $\mathbb{Q}$, there still is a modularity result over some extension:

Theorem 29 [Shioda and Inose 1977, Theorem 6]. Upon increasing the base field, the $\zeta$-function of a singular $K 3$ surface $X$ splits into one-dimensional factors. Then the L-function of the transcendental lattice factors as

$$
L(T(X), s)=L\left(\psi^{2}, s\right) L\left(\bar{\psi}^{2}, s\right),
$$

where $\psi$ is the Hecke character associated to an elliptic curve with CM in K. Here one can choose the elliptic curve E identified with the transcendental lattice $T(S)$ under the map

$$
\left(\begin{array}{cc}
2 a & b \\
b & 2 c
\end{array}\right) \mapsto \tau=\frac{-b+\sqrt{b^{2}-4 a c}}{2 a} \mapsto E=\mathbb{C} /(\mathbb{Z}+\tau \mathbb{Z}) .
$$

Thanks to this result, we are able to derive the relevant properties of $a_{\mathfrak{p}}$ to apply our previous techniques. We will need the following lemma:

Lemma 30. In the above notation, $a_{\mathfrak{p}} \in K$. Moreover $a_{\mathfrak{p}}$ takes the shape of Example 5 and $p$ splits into primes of order two in $\mathrm{Cl}(K)$ :

$$
p^{2}=\alpha_{\mathfrak{p}} \cdot \bar{\alpha}_{\mathfrak{p}}=x^{2}+D y^{2}, \quad a_{\mathfrak{p}}= \pm 2 x
$$

Remark 31. By inspection, Lemma 30 does not require NS $(X)$ to be rational over $\mathbb{F}_{\mathfrak{p}}$, but only $p$ to split in $K$ and $\mathfrak{p} \mid p$. In our special case where $\operatorname{NS}(X)$ is fully defined over $\mathbb{F}_{\mathfrak{p}}$, the proof of Proposition 28 will ultimately show that $p$ splits into principal primes in $\mathrm{Cl}(d)$.

Proof of Lemma 30. From the Weil conjectures we know that

$$
a_{\mathfrak{p}}=\alpha_{\mathfrak{p}}+\bar{\alpha}_{\mathfrak{p}} \text { with }\left|\alpha_{\mathfrak{p}}\right|=p .
$$

Here $\alpha_{\mathfrak{p}}, \bar{\alpha}_{\mathfrak{p}}$ are algebraic integers, complex conjugate in an imaginary quadratic extension of $\mathbb{Q}$ since $a_{\mathfrak{p}} \in \mathbb{Z}$. We have to show that this quadratic field is $K$. In the present situation we know the $\zeta$-function of $X$ over some extension of $L$ by Theorem 29. As a result of increasing the ground field, the eigenvalues $\psi(\mathfrak{P})^{2}, \overline{\psi(\mathfrak{P})}^{2}$ of Frobenius at a prime $\mathfrak{P}$ above $\mathfrak{p}$ agree with some power of $\alpha_{\mathfrak{p}}, \bar{\alpha}_{\mathfrak{p}}$. Since $\psi(\mathfrak{P}) \in K \backslash \mathbb{Q}$ and $\alpha_{\mathfrak{p}}$ is quadratic over $\mathbb{Q}$, this implies that $\alpha_{\mathfrak{p}} \in K$. It follows that $a_{\mathfrak{p}}$ has exactly the same shape as $a_{p}$ in Example 5. In fact, we deduce from (20) that

$$
p^{2}=\alpha_{\mathfrak{p}} \cdot \bar{\alpha}_{\mathfrak{p}}=x^{2}+D y^{2}, \quad \text { where } a_{\mathfrak{p}}=\alpha_{\mathfrak{p}}+\bar{\alpha}_{\mathfrak{p}}= \pm 2 x
$$


This is to say that the prime factors of $p$ in $K$ become principal upon squaring.

Thanks to Lemma 30 we can continue exactly along the lines of the previous sections to complete the proof of Proposition 28. We distinguish two cases:

If $L=K$, then at every good split prime $\mathfrak{p}$ in $K$, we have $\rho\left(X / \mathbb{F}_{\mathfrak{p}}\right)=20$. Hence the arguments from the previous sections carry over except for Lemma 22 . That is, $d$ has class number one, but imprimitive $T(X)$ occurs.

If $L \neq K$, then Proposition 14 tells us that all the primes that split in both $K$ and $L$ are principal. Hence $K$ has class number one or two. By the argumentation of Section 8, all these $p$ are principal in $\mathrm{Cl}(d)$ as well (as mentioned in Remark 31). Hence, $d$ has class number one or two. In the latter case, $L K=H(d)$ by class field theory.

Remark 32. For many K 3 surfaces with Picard rank 20 over a quadratic extension, we know a model over $\mathbb{Q}$. Most of these models arise through the Shioda-Inose fibration [Inose 1978; Schütt 2007b] or through extremal elliptic surfaces [Beukers and Montanus 2008; Schütt 2007a]. It is an open question whether all K3 surfaces with Picard rank 20 over a quadratic extension (or more generally with discriminant $d$ of class number two) might have a model over $\mathbb{Q}$.

\section{Singular K3 surfaces over number fields}

We conclude the paper with an application of our techniques to general singular K3 surfaces. We will derive an explicit obstruction for the field of definition of the surface and that of its Néron-Severi group. First we recall a possible field of definition:

Lemma 33. Let $X$ be a singular $K 3$ surface of discriminant $d$. Then $X$ has a model over the ring class field $H(d)$.

A model was given in [Schütt 2007b, proof of Proposition 10], based on Inose's fibration [1978] (compare [Shioda 2006]). Elkies [2007] announced another model.

In general, the field $H(d)$ need not be the optimal field of definition. In fact, there are examples of singular K3 surfaces over $\mathbb{Q}$ where $H(d)$ has degree 16 or 24 over $K$. The question arises how far one can possibly descend $X$, starting from $H(d)$. Shimada [2009] (for fundamental $d$ ) and the author [Schütt 2007b] (in full generality) derived the following condition in terms of lattice:

$$
\left\{T\left(X^{\sigma}\right) ; \sigma \in \operatorname{Aut}(\mathbb{C} / K)\right\}=\text { genus of } T(X) .
$$

In Section 2, we used this to the following effect: If $X$ is defined over $\mathbb{Q}$, then the genus of $T(X)$ consists of a single class, that is, $\mathrm{Cl}(T(X)) \cong(\mathbb{Z} / 2)^{g}$. 
To rephrase (21) in terms of class field theory, denote the degree of primitivity of $T(X)$ by $m$. Write $d=m^{2} d^{\prime}$, so that we can identify

$$
\mathrm{Cl}(T(X)) \cong \mathrm{Cl}\left(d^{\prime}\right) .
$$

Let $G=\mathrm{Cl}\left(d^{\prime}\right)$ [2], the two-torsion subgroup of $\mathrm{Cl}\left(d^{\prime}\right)$, and $M$ the fixed field of $G$ in the abelian Galois extension $H\left(d^{\prime}\right) / K$.

Lemma 34. Let $X$ be a singular $K 3$ surface over some number field $L$. In the above notation,

$$
M \subset K L .
$$

So far, this was the only known obstruction to fields of definition of singular K3 surfaces. The only drawback of Lemma 34 is that it fails to measure the degree of primitivity of $T(X)$. For this reason, Theorem 2 provides a major improvement: By providing bounds on the discriminant $d$, it also implies restrictions on the degree of primitivity. We shall now apply the techniques from the previous sections to prove Theorem 2.

Proof of Theorem 2. Without loss of generality, we can assume that $L$ contains $K$. We consider all those good primes $p$ that split completely in $L$. Let $\mathfrak{p} \mid p$ in $L$. Then $\mathbb{F}_{\mathfrak{p}}=\mathbb{F}_{p}$ and $\rho\left(X / \mathbb{F}_{p}\right)=20$. Hence we can apply the Artin-Tate conjecture at $\mathfrak{p}$. As in the previous section, Lemma 30 guarantees that $a_{\mathfrak{p}}$ has the shape of Example 5 and $p$ splits into prime ideals of order two in $\mathrm{Cl}(K)$. By the argumentation of Sections 6-8, $p$ is not only represented by the principal class of $\mathrm{Cl}(K)$, but also of $\mathrm{Cl}(d)$. Hence, by class field theory, $L$ has to contain the ring class field $H(d)$.

Since there are only limited possibilities for the Galois action on the NéronSeveri lattice of a singular K3 surface (or on any lattice of given rank), Theorem 2 provides us with a direct proof of the following finiteness result due to Shafarevich. For best efficiency, Theorem 2 should be combined with Lemma 34.

Theorem 35 [Shafarevich 1996]. Let $n \in \mathbb{N}$. Then

$$
\#\{\text { singular } K 3 \text { surface X over } L ;[L: \mathbb{Q}] \leq n\}_{/ \cong}<\infty .
$$

Remark 36. Similar results can be established for other modular surfaces, for instance for singular abelian surfaces [Shioda and Mitani 1974]. In that particular case, they would also follow from the cohomological structure (see Remark 11).

\section{Acknowledgements}

The author would like to thank N. Elkies, I. Shimada and T. Shioda for many stimulating discussions. Funding from DFG under research grant Schu 2266/2-2 is gratefully acknowledged. 


\section{References}

[Artin and Swinnerton-Dyer 1973] M. Artin and H. P. F. Swinnerton-Dyer, "The Shafarevich-Tate conjecture for pencils of elliptic curves on K3 surfaces”, Invent. Math. 20 (1973), 249-266. MR 54 \#5240 Zbl 0289.14003

[Beukers and Montanus 2008] F. Beukers and H. Montanus, "Explicit calculation of elliptic fibrations of K3-surfaces and their Belyi-maps", pp. 33-51 in Number theory and polynomials, edited by J. McKee and C. Smyth, London Math. Soc. Lecture Note Ser. 352, Cambridge Univ. Press, Cambridge, 2008. MR 2009j:14011

[Elkies 2007] N. D. Elkies, "The maximal Mordell-Weil rank of an elliptic K3 surface over $\mathbb{Q}(t)$ ", talk at conference on Birational Automorphisms of Compact Complex Manifolds and Dynamical Systems at Nagoya University, 28 August 2007.

[Elkies 2008a] N. D. Elkies, "Mordell-Weil generators for singular Shioda-Inose surfaces over $\mathbb{Q} "$, preprint, 2008, Available at http://www.math.harvard.edu/ elkies/K3_20SI.html.

[Elkies 2008b] N. D. Elkies, "Shimura curve computations via K3 surfaces of Néron-Severi rank at least 19", pp. 196-211 in Algorithmic number theory, edited by A. J. van der Poorten and A. Stein, Lecture Notes in Comput. Sci. 5011, Springer, Berlin, 2008. MR 2467847 Zbl 05279287

[Elkies and Schütt 2008] N. D. Elkies and M. Schütt, "K3 surfaces and modular forms", preprint, 2008. arXiv 0809.0830

[Hall 1971] M. Hall, Jr., "The Diophantine equation $x^{3}-y^{2}=k$ ", pp. 173-198 in Computers in number theory (Oxford, 1969), edited by A. O. L. Atkin and B. J. Birch, Proc. Sci. Res. Council Atlas Sympos. 2, Academic Press, London, 1971. MR 48 \#2061 Zbl 0225.10012

[Hulek and Verrill 2005] K. Hulek and H. A. Verrill, "On the motive of Kummer varieties associated to $\Gamma_{1}(7)$ ", J. Math. Kyoto Univ. 45:4 (2005), 667-681. supplement to R. Livné and N. Yui, "The modularity of certain non-rigid Calabi-Yau threefolds", J. Math. Kyoto Univ. 45:4 (2005), 645665. MR 2007b:11092 Zbl 1106.14023

[Inose 1978] H. Inose, "Defining equations of singular $K 3$ surfaces and a notion of isogeny", pp. 495-502 in Proceedings of the International Symposium on Algebraic Geometry (Kyoto, 1977), edited by M. Nagata, Kinokuniya Book Store, Tokyo, 1978. MR 81h:14021 Zbl 0411.14009

[Liu et al. 2005] Q. Liu, D. Lorenzini, and M. Raynaud, "On the Brauer group of a surface", Invent. Math. 159:3 (2005), 673-676. MR 2005k:14036 Zbl 1077.14023

[Livné 1995] R. Livné, "Motivic orthogonal two-dimensional representations of $\mathrm{Gal}(\overline{\mathbf{Q}} / \mathbf{Q})$ ", Israel J. Math. 92:1-3 (1995), 149-156. MR 96m:11050 Zbl 0847.11035

[Milne 1975] J. S. Milne, “On a conjecture of Artin and Tate”, Ann. of Math. (2) 102:3 (1975), $517-$ 533. See also his addendum to this article at http://www.jmilne.org/math/articles/add/addendaA.pdf. MR 54 \#2659 Zbl 0343.14005

[Miranda and Persson 1986] R. Miranda and U. Persson, "On extremal rational elliptic surfaces", Math. Z. 193:4 (1986), 537-558. MR 88a:14044 Zbl 0652.14003

[Piatetskiü-Shapiro and Shafarevich 1971] I. I. Piatetskiǔ-Shapiro and I. R. Shafarevich, "Torelli’s theorem for algebraic surfaces of type K3", Izv. Akad. Nauk SSSR Ser. Mat. 35 (1971), 530-572. In Russian. MR 44 \#1666 Zbl 0219.14021

[Ribet 1977] K. A. Ribet, "Galois representations attached to eigenforms with Nebentypus", pp. 17-51 in Modular functions of one variable (Bonn, 1976), edited by J.-P. Serre and D. B. Zagier, Lecture Notes in Math. 601, Springer, Berlin, 1977. MR 56 \#11907 Zbl 0363.10015

[Schütt 2006] M. Schütt, Hecke eigenforms and the arithmetic of singular K3 surfaces, dissertation, Universität Hannover, 2006. 
[Schütt 2007a] M. Schütt, "Elliptic fibrations of some extremal K3 surfaces", Rocky Mountain J. Math. 37:2 (2007), 609-652. MR 2008c:14047

[Schütt 2007b] M. Schütt, "Fields of definition of singular K3 surfaces", Commun. Number Theory Phys. 1:2 (2007), 307-321. MR 2008g:14060 Zbl 1157.14308

[Schütt 2008] M. Schütt, "Arithmetic of a singular K3 surface”, Michigan Math. J. 56:3 (2008), 513-527. MR 2009k:11106 Zbl 1163.14022

[Schütt 2009] M. Schütt, “CM newforms with rational coefficients”, Ramanujan J. 19:2 (2009), 187-205. MR 2511671 Zbl 05633530

[Schütt and Schweizer 2007] M. Schütt and A. Schweizer, "On the uniqueness of elliptic K3 surfaces with maximal singular fibre”, preprint, 2007. arXiv 0712.3873

[Schütt and Top 2006] M. Schütt and J. Top, "Arithmetic of the [19, 1, 1, 1, 1, 1] fibration", Comment. Math. Univ. St. Pauli 55:1 (2006), 9-16. MR 2007m:14053 Zbl 1125.14022

[Shafarevich 1996] I. R. Shafarevich, "On the arithmetic of singular K3-surfaces”, pp. 103-108 in Algebra and analysis (Kazan, 1994), edited by M. M. Arslanov et al., de Gruyter, Berlin, 1996. MR 98h:14041 Zbl 0947.14020

[Shimada 2009] I. Shimada, "Transcendental lattices and supersingular reduction lattices of a singular K3 surface”, Trans. Amer. Math. Soc. 361:2 (2009), 909-949. MR 2009m:14055 Zbl 05518627

[Shioda 1990] T. Shioda, "On the Mordell-Weil lattices", Comment. Math. Univ. St. Paul. 39:2 (1990), 211-240. MR 91m:14056 Zbl 0725.14017

[Shioda 1994] T. Shioda, "On the rank of elliptic curves over $\mathbf{Q}(t)$ arising from $K 3$ surfaces", Comment. Math. Univ. St. Paul. 43:1 (1994), 117-120. MR 95c:14041 Zbl 0815.14022

[Shioda 2003] T. Shioda, "The elliptic $K 3$ surfaces with with a maximal singular fibre", C. R. Math. Acad. Sci. Paris 337:7 (2003), 461-466. MR 2004j:14046 Zbl 1048.14017

[Shioda 2005] T. Shioda, "On K3 surfaces defined over Q", Comment. Math. Univ. St. Pauli 54:1 (2005), 87-88. Correction to [Shioda 1994]. MR 2006b:14065 Zbl 0815.14022

[Shioda 2006] T. Shioda, "Kummer sandwich theorem of certain elliptic K3 surfaces", Proc. Japan Acad. Ser. A Math. Sci. 82:8 (2006), 137-140. MR 2008b:14064 Zbl 1112.14044

[Shioda and Inose 1977] T. Shioda and H. Inose, "On singular K3 surfaces", pp. 119-136 in Complex analysis and algebraic geometry, edited by W. L. B. Jr. et al., Iwanami Shoten, Tokyo, 1977. MR 56 \#371 Zbl 0374.14006

[Shioda and Mitani 1974] T. Shioda and N. Mitani, "Singular abelian surfaces and binary quadratic forms", pp. 259-287 in Classification of algebraic varieties and compact complex manifolds, edited by H. Popp, Lecture Notes in Math. 412, Springer, Berlin, 1974. MR 52 \#3174 Zbl 0302.14011

[Tate 1965] J. T. Tate, "Algebraic cycles and poles of zeta functions", pp. 93-110 in Arithmetical Algebraic Geometry (West Lafayette, IN, 1963), edited by O. F. G. Schilling, Harper \& Row, New York, 1965. MR 37 \#1371 Zbl 0213.22804

[Tate 1966] J. Tate, "On the conjectures of Birch and Swinnerton-Dyer and a geometric analog", pp. 415-440 in Sém. Bourbaki 1965/66 (Exposé 306), edited by N. H. K. A. Grothendieck, 1966. Reprinted as pages 189-214 of Dix exposés sur la cohomologie des schemas, North-Holland, Amsterdam, 1968. MR 1610977 Zbl 0199.55604

[Tate 1974] J. T. Tate, “The arithmetic of elliptic curves”, Invent. Math. 23 (1974), 179-206. MR 54 \#7380 Zbl 0296.14018

[Top and Yui 2007] J. Top and N. Yui, "Explicit equations of some elliptic modular surfaces", Rocky Mountain J. Math. 37:2 (2007), 663-687. MR 2008c:14049 Zbl 1140.14036 
[Weinberger 1973] P. J. Weinberger, "Exponents of the class groups of complex quadratic fields", Acta Arith. 22 (1973), 117-124. MR 47 \#1776

Communicated by János Kollár

Received 2009-07-21 Revised 2009-11-14 Accepted 2009-12-31

schuett@math.uni-hannover.de Institut für Algebraische Geometrie, Leibniz Universität Hannover, Welfengarten 1, 30167 Hannover, Germany http://www.iag.uni-hannover.de/ schuett/ 